\title{
仿人背抱式移乘护理机器人背负运动轨迹规划与 舒适性分析
}

\author{
刘玉金金 ${ }^{1,2,3}$ 郭士杰 ${ }^{1,2,3}$ 陈贵亮 ${ }^{2,3}$ 刘今越 2,3 甘中学 4
}

(1. 河北工业大学省部共建电工装备可靠性与智能化国家重点实验室 天津 300130;

2. 河北省机器人感知与人机融合重点实验室 天津 300130;

3. 河北工业大学机械工程学院 天津 300130;

4. 复旦大学工程与应用技术研究院 上海 200433)

摘要: 护理过程中, 失能半失能人群的移乘服务给护理人员带来过重的身体负担, 导致多数养老机构的护理人员患有慢性腰椎疾病。 但目前以移位机为代表的移乘辅助器械大都使用不便, 耗时费力, 普及率较低。为实现下肢失能老人在生活器具间安全且方便地移乘, 研制了一款仿人背抱式移乘护理机器人。该机器人采用三自由度胸靠支撑被护理人的胸部, 通过模仿人背人的动作设计, 把被护理人 背/抱起进行移乘。从研究人背人的动作特征入手, 分析了影响移乘舒适性的因素, 设计了机器人构型, 建立了人机系统的力学模型, 通过运动学及动力学解析优化了机器人机构及运动轨迹, 明确了机器人的工作空间及其特有的运动特性。对 8 名不同身高体重的试验 对象的试验结果显示, 所研制的机器人可以较为安全舒适地完成仿人背抱动作并在不同生活器具间进行移乘服务。人机之间相互作用 力的大小及其作用部位是影响舒适感的直接因素, 因此可以通过调整胸靠的运动轨迹, 实现不同身高体重的被护理人的舒适移乘。

关键词: 护理机器人; 仿人背抱动作; 移乘搬运; 舒适性。

中图分类号: TP242

\section{Bionic Motion Planning and the Analysis for Human Comfort of a Piggyback Nursing-care Robot for Transfer Tasks}

\author{
LIU Yuxin ${ }^{1,2,3}$ GUO Shijie G, $^{1,3}$ CHEN Guiliang, ${ }^{2,3}$ LIU Jinyue ${ }^{2,3}$ GAN Zhongxue ${ }^{4}$ \\ (1. State Key Laboratory of Reliability and Intelligence of Electrical Equipment, Hebei University \\ of Technology, Tianjin 300130;
}

2. Hebei Key Laboratory of Robot Sensing and Human-robot Interaction, Tianjin 300130;

3. School of Mechanical Engineering, Hebei University of Technology, Tianjin 300130;

4. Academy for Engineering \& Technology, Fudan University, Shanghai 200433)

\begin{abstract}
Patient transfer, such as transferring a bedridden care receiver from a bed to a wheelchair or a pedestal pan and back, is one of the most physically challenging tasks in nursing care. It is the direct reason that causes most of the caregivers working in care facilities to suffer from chronic lumbar diseases. A few kinds of transfer lifts have been commercialized to solve the problem, but they are rarely used because they are time-consuming and difficult to handle. Imitating the motion when a person holds another person on his/her back, a prototype nursing-care assistant robot is developed to conduct the patient transfer. Based on the measurement of human motions including back-chest touching, leaning forward, holding up, moving and putting down, the key factors that may influence safety and comfort of the patient is analyzed and a new type of robot is designed. The robot consists of a chest holder which has three degrees of freedom and moves like a human back. This work states the details of motion design and related kinematic/dynamic analysis of the robot and demonstrate its effect on patient transfer. Experiments has been performed on eight subjects and it is demonstrated that the robot can transfer a subject from a bed to a chair and back. The force between the care receiver and the robot during transfer is the direct factor affecting the feeling of the care receiver. The robot can adapt to various care receivers with different height and weight by adjusting the motion trajectory of the chest holder.
\end{abstract}

Key words: nursing-care robot; bionic piggyback motion; patient transfer; comfortableness

* 国家重点研发计划(2016YFE0128700, 2017YFB1301002)、河北省重点 研发计划(18211816D)和河北省自然科学基金(E2017202270)资助项目。 20190827 收到初稿, 20191211 收到修改稿 


\section{0 前言}

随着人口老龄化加剧, 失能/半失能人群数量增 加 ${ }^{[1]}$, 护理人手不足及护理人员身体负担过重等问 题日益凸显 ${ }^{[2-5]}$ 。对失能/半失能人群的护理包括饮 食服务、卫生清洁、健康管理、精神慰藉、移乘服 务等多个方面, 其中对护理人员身体负担最重的是 移乘服务。移乘服务是指协助或搬运行动不便的被 护理人完成其在床、轮椅、马桶、沙发等生活器具 之间的移乘。作为重体力劳动, 它是导致绝大部分 护理人员患有慢性腰椎疾病的直接原因 ${ }^{[6]}$; 在家庭, 它是制约老老护理(老年夫妇间的相互照料、老龄子 女对父母的照料等)的主要因素。

目前常见的移乘搬运设备可分为直接方式与间 接方式两大类。直接方式是指床与轮椅采用一体化 设计 ${ }^{[7-8]}$, 床或床的一部分可直接折叠成轮椅, 实现 被护理人在室内的移动; 间接方式是指在不同生活 器具间采用某种移乘装置进行搬运。由于直接移乘 设备一人一床, 成本高, 且无法实现床与马桶之间 的移乘，因此应用较少。目前常见的移乘搬运设备 以间接方式为主, 其中电动移位机因结构简单、成 本低廉, 最为常用 ${ }^{[9]}$ 。电动移位机大都采用吊具将 被护理人悬吊起来进行移动, 但存在吊具置入被护 理人身下困难、费时费力、舒适性差、易造成二次 伤害等问题。鉴于此, 研发安全可靠的智能移乘搬 运设备一直是护理器械领域的一个研究热点 ${ }^{[10-11]}$ 。

日本松下电器公司研制的 Transfer Assist Robot ${ }^{[12]}$ 采用双臂构型, 通过类似公主抱的动作, 可 在一名护理人员操作下实现被护理人进行在床与床 之间的移乘。但该机器人存在整体尺寸过大、无法 实现床到轮椅的移乘、舒适性差等问题。日本 RIKEN 研发的 RI-MAN、RIBA ${ }^{[13-15]}$ 等双臂移乘护 理机器人, 全身具有触觉感知功能, 可通过与人一 样灵活的双臂, 将被护理人抱起并在床与轮椅间进 行移乘; 美国 Hstar Technologies 公司设计的双臂护 理机器人 RoNA ${ }^{[16]}$, 通过其手臂前掌与被护理人接 触部位的传送带机构, 可将平躺在床上的被护理人 以剪切力最小的方式抱起并进行移动。但拟人双臂 护理机器人存在结构复杂、操作繁琐、成本过高、 安全性不足等问题，制约了其产业化推广。相比拟 人双臂护理机器人, 丰田汽车公司 ${ }^{[17]}$ 和富士机械制 造有限公司 ${ }^{[18]}$ 研制的前抱式移乘护理机器人, 通过 一个 2 自由度的背负装置, 实现了被护理人在床、 轮椅、马桶之间的移乘。此类机器人结构简单、成
本较低, 但较少的自由度限制了机器人末端动作的灵 活性，存在舒适性差、易引起被护理人压痛等问题。

本文提出一种新型仿人背抱式移乘护理机器人 构型, 以实现下肢失能的被护理人在床、轮椅、沙 发、则所马桶等生活器具间的移乘, 如图 1 所示。 在日常护理服务中, 特别是一对一的护理服务中, 面对体重较大的被护理人或需要移动较长距离时, 护理人员常采用背负方式进行移乘服务。背负过程 中，护理人员的双臂用于维持被护理人姿态，并支 撑部分体重, 而被护理人的大部分体重由护理人员 背部承受。背负动作虽然对护理人员的身体负担重, 但具有用时短、姿势较为稳定等优点。因此, 本研 究从分析人背人的运动特征入手, 通过模仿人背人 的动作, 设计了机器人构型和动作模式并研制了样 机。试验结果显示, 所设计的机器人能够较为舒适 地将试验对象从登子移乘到床、沙发、轮椅等生活 器具，具有较高的实用性。

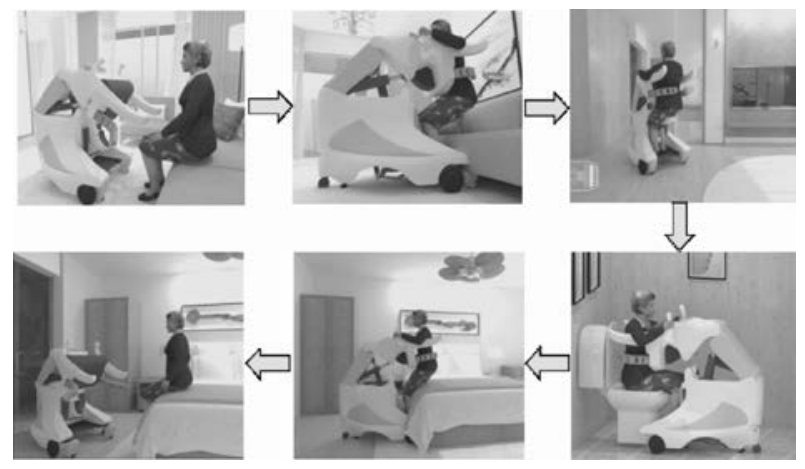

图 1 仿人背抱式护理机器人移乘过程

\section{1 人背人动作分析}

\section{1 人背人试验设计}

图 2 为试验过程中拍摄的人背人动作照片。本 研究利用 OptiTrack(V1.0)运动捕捉系统采集背负过 程中被背人的动作。为此, 依据 ISO/IEC19774Human animation(H-Anim)标准人体骨架模型 ${ }^{[19]}$, 选 取模型中的 “ cervicale”、“ rib10_midspine”、 “waist_preferred_post” 作为标记点进行了标记, 如 图 3 所示。被背人端坐于登子上, 登子的高度可由 被背人根据自身身高及主观感受进行调整。试验动 作设计为背负人将坐在登子上的被背人背起，并步 行移动。

本研究选择了身高、体重不同的 5 名志愿者作 为被背人(表 1 ), 选择 1 名身高 $1700 \mathrm{~mm}$, 体重 $80 \mathrm{~kg}$ 的 27 岁男性志愿者作为背负人进行了试验。 为避免腹部饱涨对背负动作产生影响, 试验在上午 
进行, 要求被背人和背负人试验前日不饮酒, 充分 休息, 试验开始前两小时内禁止饮食。此外, 为避 免由于连续试验造成的身体疲劳对背负动作产生影 响, 试验过程中, 要求每组背负动作后被背人和背 负人都休息 $5 \mathrm{~min}$, 方可进行下一组试验。

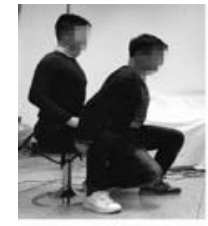

(a)

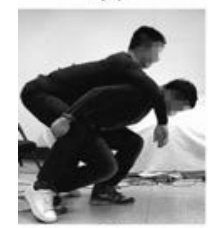

(d)

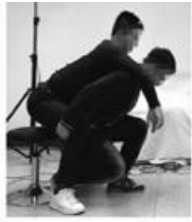

(b)

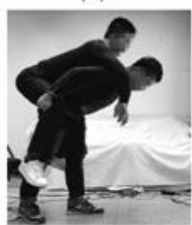

(e)

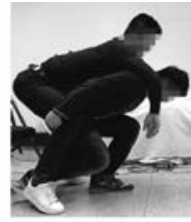

(c)

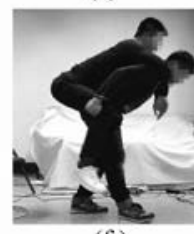

(f)
图 2 背负运动过程
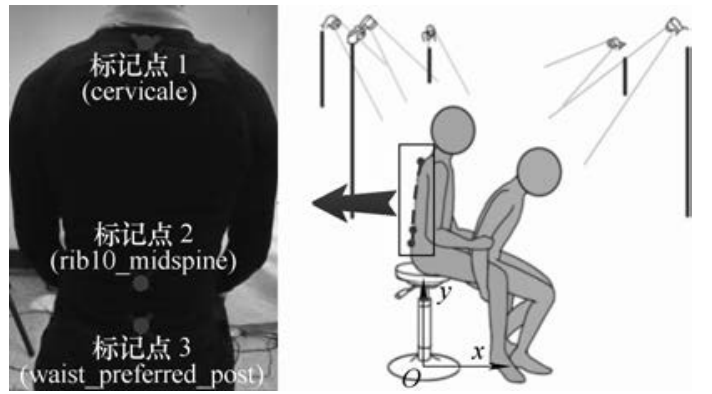

图 3 人背人动作试验布置

表 1 被背人信息表

\begin{tabular}{ccccc}
\hline & 身高/mm & 体重/kg & 年龄 & 性别 \\
\hline 被背人 1 & 1550 & 67 & 28 & 男 \\
被背人 2 & 1710 & 65 & 27 & 男 \\
被背人 3 & 1730 & 60 & 24 & 男 \\
被背人 4 & 1750 & 63 & 26 & 男 \\
被背人 5 & 1780 & 67 & 26 & 男 \\
\hline
\end{tabular}

\section{2 人背人动作分析}

人背人运动过程如图 2 所示, 其中背起动作包 括 “靠近”、“升抬”、“姿势调整”三个阶段。靠近 过程中, 被背人端坐于発子上, 当背负人下蹲至其 肩膀略低于被背人肩膀时, 被背人的躯干前倾, 直 至其胸部完全贴附于背负人的背部; 升抬时, 背负 人首先保持其靠近时的腿部姿势, 其躯干围绕髋关 节前倾, 待调整两人重心位于其双脚之上后, 开始 站起, 完成升抬动作。升抬过程中, 背负人与被背 人的背部角度变化较小, 待背负人的下肢完全站立 后, 其躯干开始调整姿势, 为 “背负行走” 做准备。 在此, 背负人姿势调整主要是指其背部绕髋关节向
后回转, 减小胸部前倾角度。

以身高 $1730 \mathrm{~mm}$, 体重 $60 \mathrm{~kg}$ 的被背人为例, 背负过程中的标记点运动轨迹如图 4 所示。图中, 将被背人的背部等效为刚性杆, 以登子在地面的投 影的中心作为原点建立坐标系, 其横轴/纵轴分别为 各标记点的水平/坚直方向位移, 其中, 通过以被背 人身高为分母对坚直方向位移进行无量纲化。被背人 躯干的绝对角度变化如图 5 所示, 当被背人处于前倾 阶段时, 其躯干趋于水平, 绝对角度减小; 而在升抬、 稳定背负阶段过程中, 躯干绝对角度变化相对较小。 结合背负过程中被背人的主观感受, 背负人与被背人 间相对位置、背负人姿态以及由姿态改变引发的两者 间作用力的变化都会影响被背人的舒适; 且在被背人 重力作用下, 背负人与被背人间的作用力随姿态而改 变, 当被背人的躯干角度在 $5^{\circ} \sim 20^{\circ}$ 的范围内时, 被背人腋下、胸部、腹部等接触部位的受力均在其可 接受范围内, 被背人感受 “舒适”。

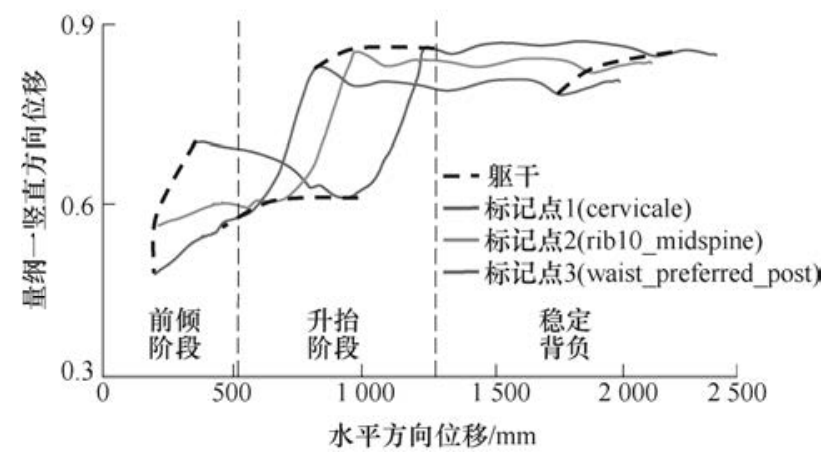

图 4 被背人躯干运动轨迹

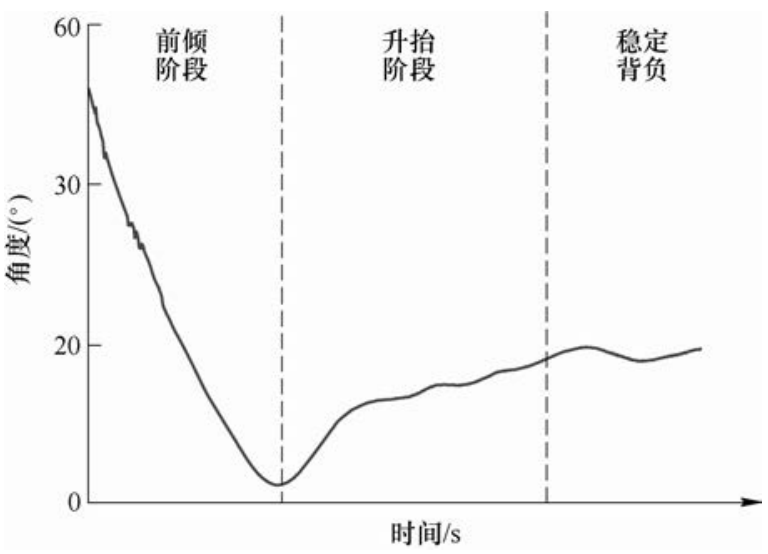

图 5 躯干角度变化

5 位被背人背部标记点各自的最小轨迹范围如 图 6 所示, 其中, 每个标记点的坚直方向位移分别 以各自对应的被背人身高为分母进行了无量纲化。 此最小轨迹范围对机器人的工作空间设计具有十分 重要的作用。 


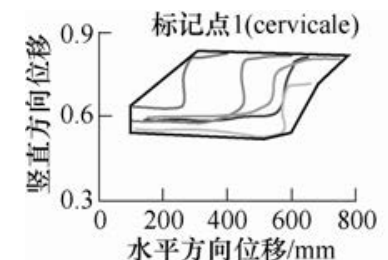

(a)
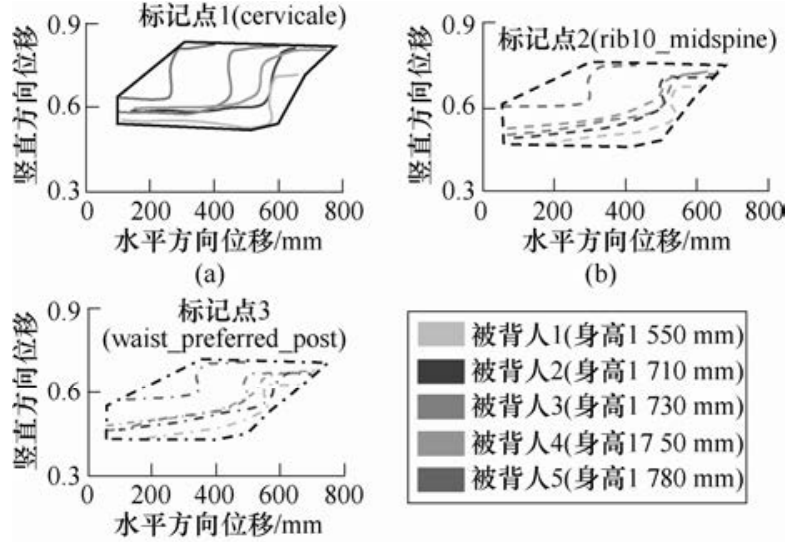

(b)

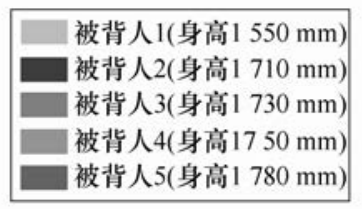

(c)

图 6 被背人各标记点最小轨迹范围

人背人试验结果表明, 在背负人与被背人的相 对位置基本保持不变的情况下, 被背人的受力随着 背负人姿态的改变, 直接影响被背人的主观感受。 此外, 为确保移乘过程中被背人的舒适性, 机器人 也应具有合理的动作。参照人背人的运动特性, 机 器人末端的胸靠应具有包括水平、坚直移动及旋转 的 3 个自由度, 如图 7 所示。

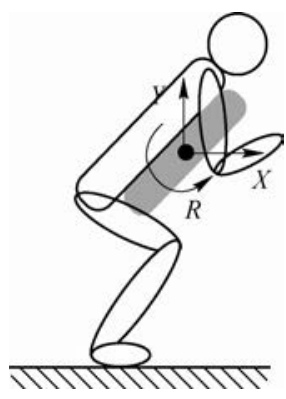

图 7 机器人胸靠概念图

\section{2 机构设计}

\section{1 机构总体方案}

依据人背人运动特征所设计的机器人主要由背 人机构与移动部分构成, 其构型如图 8 所示。机器人 的背人结构由两条动臂及胸靠组成, 其中胸靠的两侧 对称安装有柔性手臂与腋下支撑装置。机器人胸靠在 两动臂作用下可模仿人背人动作, 通过支撑被护理人 的胸部及腋下将其抱起。机器人的移动部分除两个驱 动轮和一个从动轮外, 还包括有可自动执行的踏板、 防止机器人翻倒装置以及臀部支撑装置。在机器人移 运过程中, 被护理人坐于慰部支撑上, 减小了其胸部 与腋下的受力, 提高了舒适性。此外, 为减轻机器人 整体重量, 使其结构紧凑, 机身采用析架结构搭建。 该机器人的外观尺寸为: 机身宽度 $755 \mathrm{~mm}$, 纵深 $1160 \mathrm{~mm}$, 胸靠可达最大高度 $1964 \mathrm{~mm}$ 。

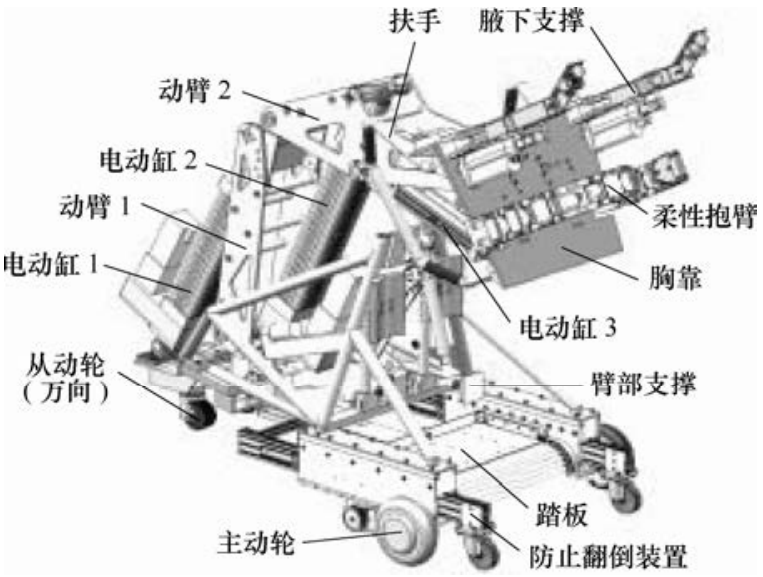

图 8 仿人背抱式移乘护理机器人结构

\section{2 背人机构设计}

机器人背人结构采用混联结构, 以电动缸作为驱 动元件, 实现了胸靠与人背人相似的运动轨迹。该胸 靠作为机器人的末端具有旋转、水平/坚直位移 3 个自 由度, 通过 3 个电动缸的伸缩运动实现, 如图 9 所示。 其中, 电动缸 1 首尾铰接于动臂 1 与机架, 其伸缩运 动实现动臂 1 围绕动臂 1 关节的转动。动臂 2 及胸靠 的转动实现与动臂 1 实现方式一致, 不再赘述。

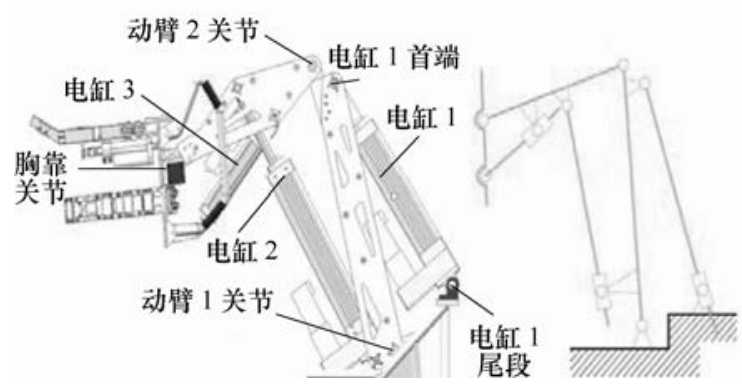

图 9 背人部分结构

为实现机器人在移乘过程中对被护理人的准确 定位，并确保移乘过程的稳定性，在胸靠的上侧对 称安装有腋下支撑，如图 10a 所示。该装置为刚性 结构, 通过多层薄弹簧钢板与胸靠连接, 连接部位 构成平行四边形机构, 配合弹簧的特性, 有效地防 止了机器人在与被护理人的硬接触, 提高了机器人 的舒适性。此外, 胸靠的中下部对称设置有柔性手 臂, 如图 10b 所示。该柔性手臂作为刚柔混合机构, 以绳索欠驱动的方式，实现了手臂的环抱动作。移 运过程中该柔性手臂通过抱紧被护理人腰部, 可有 效地防止其滑落，提高了机器人的安全性。

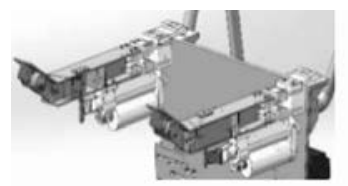

(a) 机器人腋下支䍌

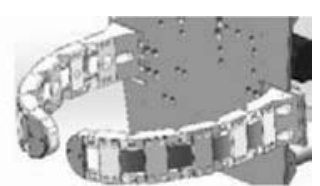

(b) 机器人柔性手臂
图 10 机器人胸靠结构 


\section{3 运动学分析}

\section{1 背人运动分析}

为实现胸靠的背人动作, 有必要依据仿生背人 的运动轨迹求解机器人背人结构的运动学参数。因 此, 以机器人的动臂 1 关节为基坐标系的原点, 动 臂关节与胸靠关节作分别为各动坐标系的原点, 建 立机器人背人机构的运动学模型, 如图 11 所示。

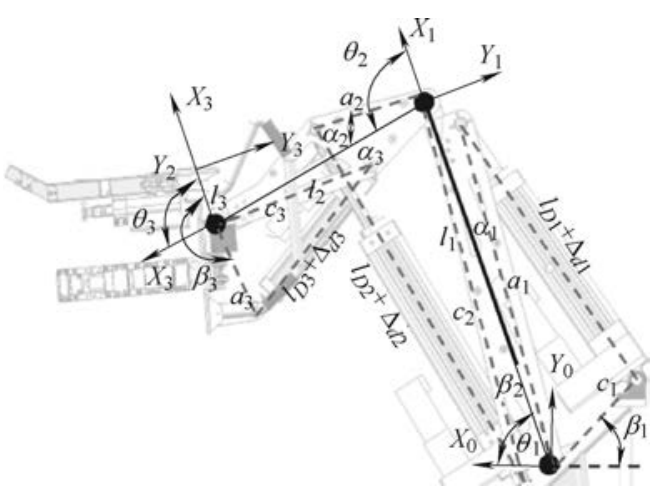

图 11 机器人背人机构运动学模型

根据 D-H 法 ${ }^{[20-21]}$, 得到胸靠最高点的坐标系相 对于基坐标系的转换矩阵如下

$$
\boldsymbol{T}=\left[\begin{array}{cccc}
c_{123} & -s_{123} & 0 & p_{x} \\
s_{123} & c_{123} & 0 & p_{y} \\
0 & 0 & 1 & 0 \\
0 & 0 & 0 & 1
\end{array}\right]=A_{1} A_{2} A_{3}
$$

通过矩阵变换, 则有

$$
\left\{\begin{array}{l}
A_{1}^{-1} T=A_{2} A_{3} \\
A_{2}^{-1} A_{1}^{-1} T=A_{3}
\end{array}\right.
$$

根据矩阵等式元素对应相等, 则有

$$
\left\{\begin{array}{l}
c_{1} p_{x}+s_{1} p_{y}-l_{1}=l_{3} c_{23}+l_{2} c_{2} \\
-s_{1} p_{x}+c_{1} p_{y}=l_{3} s_{23}+l_{2} s_{2} \\
c_{12} p_{x}+s_{12} p_{y}-c_{2} l_{1}-l_{2}=c_{3} l_{3} \\
-s_{12} p_{x}+c_{12} p_{y}+s_{2} l_{1}=s_{3} l_{3}
\end{array}\right.
$$

则背人机构各关节转角为

$$
\left\{\begin{array}{l}
\theta_{1}=\arctan \frac{p_{y}}{p_{x}}-\arctan \frac{ \pm \sqrt{(2 r l)^{2}-\left(r^{2}-l_{3}^{2}-2 l_{2} l_{3} \cos \theta_{3}\right)^{2}}}{r^{2}-l_{3}^{2}-2 l_{2} l_{3} \cos \theta_{3}} \\
\theta_{2}=\arcsin \frac{p_{y} c_{1}-p_{x} s_{1}}{\sqrt{\left(l_{2}+l_{3} c_{3}\right)^{2}+l_{3}^{2} s_{3}^{2}}}+\arctan \frac{-l_{3} s_{3}}{l_{2}+l_{3} c_{3}} \\
\theta_{3}=\arctan \frac{p_{y}}{p_{x}}
\end{array}\right.
$$

$$
\left\{\begin{array}{l}
s_{1}=\sin \theta_{1} \\
s_{12}=\sin \left(\theta_{1}+\theta_{2}\right) \\
s_{123}=\sin \left(\theta_{1}+\theta_{2}+\theta_{3}\right) \\
c_{1}=\cos \theta_{1} \\
c_{12}=\cos \left(\theta_{1}+\theta_{2}\right) \\
c_{123}=\cos \left(\theta_{1}+\theta_{2}+\theta_{3}\right)
\end{array}\right.
$$

通过机器人背人机构几何关系求解各电动缸的 伸缩量为

$$
\left\{\begin{array}{l}
\Delta d_{1}=\sqrt{a_{1}^{2}+c_{1}^{1}+2 a_{1} c_{1} \cos \left(\theta_{1}+\alpha_{1}+\beta_{1}\right)}-l_{D 1} \\
\Delta d_{2}=\sqrt{a_{2}^{2}+c_{2}^{1}+2 a_{2} c_{2} \cos \left(\theta_{2}+\alpha_{2}-\beta_{2}\right)}-l_{D 2} \\
\Delta d_{3}=\sqrt{a_{3}^{2}+c_{3}^{1}-2 a_{3} c_{3} \cos \left(\alpha_{3}+\beta_{3}\right)}-l_{D 3}
\end{array}\right.
$$

式中, $\Delta d_{1} 、 \Delta d_{2} 、 \Delta d_{3}$ 分别为电动缸 $1 / 2 / 3$ 的伸缩 量; $a_{1} 、 \alpha_{1} 、 \beta_{1} 、 c_{1} 、 l_{D 1} 、 a_{2} 、 \alpha_{2} 、 \beta_{2} 、 c_{2} 、 l_{D 2} 、$ $a_{3} 、 c_{3} 、 \alpha_{3} 、 \beta_{3} 、 l_{D 3}$ 为背人机构的相关几何尺寸, 如图 11 所示。

\section{2 工作空间}

该机器人背人机构的 D-H 参数如表 2 所示。为 使胸靠具有仿人背负的运动轨迹，并实现不同身高 的被护理人安全舒适的移乘, 该机器人胸靠应具有 合理的工作空间。在背人机构运动学分析的基础上, 得到机器人胸靠最高点的工作空间, 并根据人背人 试验中被背人的标记点与胸靠最高点之间的几何关 系，同时得到身高为 1550 1 $780 \mathrm{~mm}$ 被背者轨迹 的最小工作范围。此最小工作范围包含于胸靠的工 作空间内, 验证了所设计的机器人背人结构可满足 大多数被护理人的移乘需求，如图 12 所示。

表 2 机器人背人部分 D-H 参数

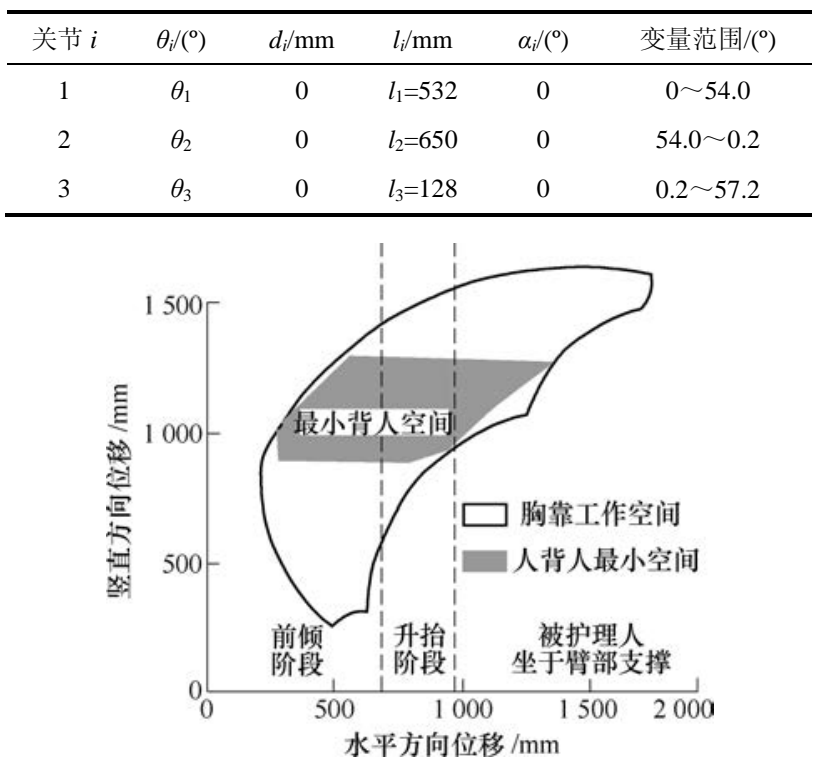

图 12 机器人胸靠工作空间 


\section{4 动力学分析与仿真}

\section{1 动力学分析}

人背人试验显示, 背负过程中背负人与被背人 间的作用力是影响舒适度的主要因素。结合机器人 实际的移乘过程, 被护理人胸部/腋下与机器人胸靠 之间的作用力随胸靠姿态的改变, 直接影响被护理 人的舒适性。因此, 将被护理人等效为质量均匀分 布的四连杆, 建立移乘搬运过程中的人机系统的力 学模型(图 13), 通过动力学解析, 分析影响被护理 人胸部/腋下压力变化的因素, 以便更好地设计机器 人的移乘搬运动作。需要指出的是, 机器人胸靠的 动作虽采用仿生背人动作设计, 却无需与人背人动 作完全一致, 因此需要寻找比人背人更为舒适的移 乘搬运动作。

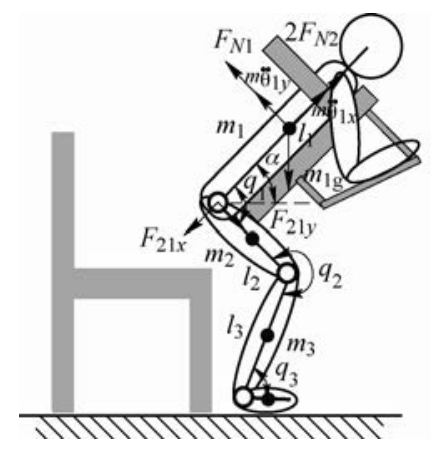

图 13 移乘过程中人机系统力学模型

移乘过程中被护理人双脚踏于机器人踏板, 其 踝关节仅存在转动。因此, 以踝关节回转中心为原 点建立全局坐标系。其中, 被护理人躯干、大腿、 小腿的质心坐标分别为

$$
\begin{gathered}
\left\{\begin{array}{l}
x_{3}=l_{3} \cos q_{3} \\
y_{3}=l_{3} \sin q_{3}
\end{array}\right. \\
\left\{\begin{array}{l}
x_{2}=l_{3} \cos q_{3}+l_{2} \cos \left(q_{3}+q_{2}\right) \\
y_{2}=l_{3} \sin q_{3}+l_{2} \sin \left(q_{3}+q_{2}\right)
\end{array}\right. \\
\left\{\begin{array}{c}
x_{1}=l_{3} \cos q_{3}+l_{2} \cos \left(q_{3}+q_{2}\right)+l_{1} \cos \left(q_{3}+q_{2}+q_{1}\right) \\
y_{1}=l_{1} \sin q_{3}+l_{2} \sin \left(q_{3}+q_{2}\right)+l_{1} \sin \left(q_{3}+q_{2}+q_{1}\right)
\end{array}\right.
\end{gathered}
$$

式中, $l_{1} 、 l_{2} 、 l_{3}$ 分别为被护理人躯干大腿、小腿 的几何长度; $q_{1} 、 q_{2} 、 q_{3}$ 为髋、膝、踝关节夹角, 其中, 躯干绝对角度 $\alpha=q_{1}+q_{2}+q_{3}-2 \pi$ 。进而得到 各连杆的动能为

$$
K_{i}=\frac{1}{2} m_{i}\left(\frac{l_{i}}{2}\right)^{2} \dot{q}^{2}+\frac{1}{2} I_{i} \dot{q}^{2}
$$

式中, $I_{i}$ 为各连杆的惯性张量。各连杆势能为

$$
P_{i}=-m_{i} g y_{i}
$$

则有被护理人的拉格朗日动力学方程

$$
L=K-P=\sum_{i=1}^{3} K_{i}+\sum_{i=1}^{3} P_{i}
$$

式中, $L$ 为该系统的拉格朗日算子, 则被护理人躯 干的拉格朗日方程为

$$
\tau_{1}=\frac{\mathrm{d}}{\mathrm{d} t} \frac{\partial L}{\partial q_{1}}-\frac{\partial L}{\partial q_{1}}
$$

式中， $\tau_{1}$ 为被护理人髋关节转矩。

机器人移乘过程中，被护理人沿平行于胸靠方 向的剪切力主要由机器人腋下支撑的支持力提供, 人机间摩擦力较小，故不考虑摩擦因素。被护理人 受躯干自身重力 $m_{1} g$ 以及下肢对躯干的作用力 $F_{21 x} 、 F_{21 y}$, 腋下支撑对被护理人腋下的支持力 $2 F_{N 2}$, 胸靠对被护理人躯干的支持力 $F_{N 1}$, 其躯干 的受力分析如图 13 所示。根据力/力矩平衡方程得 到被护理人胸部与单侧腋下受力 $F_{N 1} 、 F_{N 2}$, 则有

$$
\left\{\begin{array}{l}
F_{N 1}-m_{1} g \cos \alpha-F_{21 y}=m_{1} \ddot{q}_{1 y} \\
2 F_{N 2}-m_{1} g \sin \alpha-F_{21 x}=m_{1} \ddot{q}_{1 x} \\
\frac{l_{1}}{2} F_{N 1}-\frac{l_{1}}{2} m_{1} g \sin \alpha=\tau_{1}
\end{array}\right.
$$

式中， $F_{21 x} 、 F_{21 y}$ 作为被护理人下肢对躯干的作用 力, 可通过下肢各部分力/力矩平衡得到, 在此不进 行赘述。

\section{2 动力学仿真}

在 ADAMS 环境下对机器人的移乘过程进行动 力学仿真, 分析移乘过程中被护理人胸部、腋下接 触部位受力与姿态变化之间的关系，以及影响被护 理人受力变化的主要因素。仿真过程中, 参照人背 人试验获得的仿生背人轨迹设计机器人移乘动作, 其中将被护理人 $(1750 \mathrm{~mm}, 80 \mathrm{~kg})$ 等效为四连杆模 型, 在重力作用下其腋下、躯干作为与机器人的主 要接触部位, 分别与机器人腋下支撑、胸靠接触并 产生相互作用力。需要指出的是, 机器人的整个移 乘过程中被护理人双脚踏于机器人踏板, 其部分重 力由双脚与机器人踏板间产生的支持力承担。

机器人胸靠的运动轨迹是由水平/坚直位移以 及旋转 3 个自由度共同构成。移乘过程中的胸靠运 动轨迹与人机作用力间的关系如图 14 所示, 其中, 基于被护理人的身高与体重分别对胸靠的位移以及 人机作用力进行了无量纲化。在移乘过程中, 被护 理人躯干、腋下的受力随着胸靠姿态的变化而改变。 仿真起始, 被护理人坐于登子, 此时被护理人的大 部分体重由其腋下承受, 身干受力相对较小; 在起 
始姿态调整过程中, 胸靠运动主要表现为其角度的 改变, 此时随着胸靠绝对角度的减小(以顺时针方向 为正), 被护理人躯干前倾, 其腋下受力逐渐减小, 躯受力逐渐增加, 成为主要受力部位; 而在搬运 升抬/下降阶段, 虽然胸靠角度存在变化, 但随着胸 靠水平/坚直位移的变化, 被护理人下肢姿态改变, 其腋下与躯干受力相比上一状态变化较小, 躯干仍 为主要受力部位。

由此可见, 机器人移乘过程中的人机间作用力 随机器人胸靠的位姿而改变, 并与之对映。

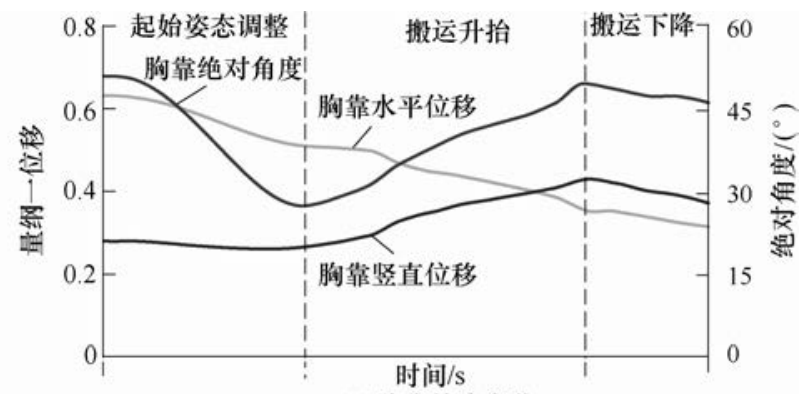

(a) 胸靠轨迹变化

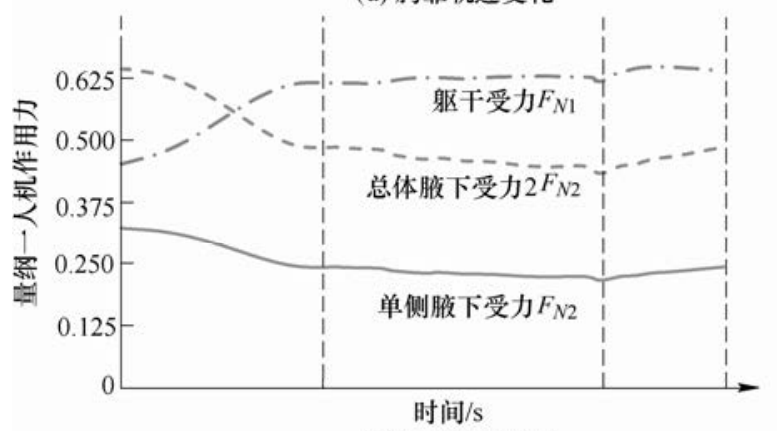

(b)人机间作用力

图 14 移乘过程胸靠轨迹与人机作用力间的关系

\section{5 样机功能试验与分析}

\section{1 机器人功能测试}

该仿人背抱式移乘护理机器人主要针对上肢健全 下肢失能人群完成不同生活器具间的移乘搬运。选取 8 名不同身高体重的试验对象(表 3), 模仿上肢健全下肢 失能的被护理人分别进行机器人移乘功能的验证。

\section{表 3 试验对象信息表}

\begin{tabular}{cccc}
\hline & 身高/mm & 体重/kg & 体重/身高 \\
\hline 试验对象 1 & 1550 & 67.0 & 0.043 \\
试验对象 2 & 1690 & 62.0 & 0.036 \\
试验对象 3 & 1730 & 63.8 & 0.037 \\
试验对象 4 & 1750 & 60.0 & 0.034 \\
试验对象 5 & 1760 & 65.0 & 0.037 \\
试验对象 6 & 1760 & 71.0 & 0.040 \\
试验对象 7 & 1780 & 69.1 & 0.039 \\
试验对象 8 & 1810 & 78.8 & 0.044 \\
\hline
\end{tabular}

以身高 $1750 \mathrm{~mm}$, 体重 $60 \mathrm{~kg}$ 的试验对象为例, 其移乘搬运过程如图 15 所示。护理之初, 在一名护 理人员协助下, 试验对象将双脚放置于机器人踏板, 并根据其主观感受调整坐姿及登子高度。待准备工作 完成后, 机器人将试验对象从登子上抱起至机器人臀 部支撑。护理过程中, 机器人胸靠逐渐靠近试验对象 的胸部, 并与其接触(图 15b)。在胸靠带动作用下, 试验对象经过前倾、升抬等过程, 被搬运至机器人的 慰部支撑(图 15c、15d)。在此过程中，臀部支撑配合 胸靠的状态自动升起。机器人将试验对象从机器人臀 部支撑移运至病床的过程如图 15e 15h 所示, 此过 程基本为图 15a 15d 的反向运动。

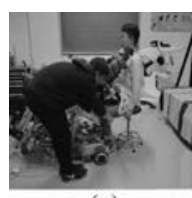

(a)

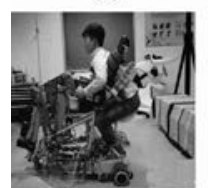

(e)

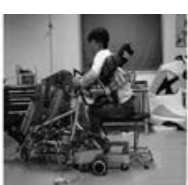

(b)

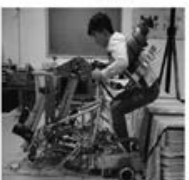

(f)

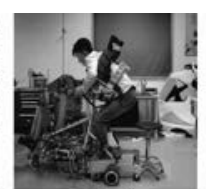

(c)

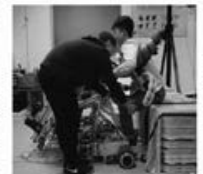

(g)

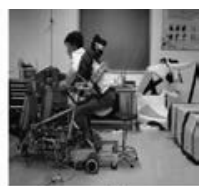

(d)

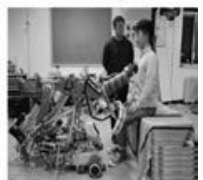

(h)
图 15 机器人移乘搬运试验

在机器人护理功能的测试试验中, 该机器人 成功地对 8 名试验对象皆完成了移乘搬运功能的 测试。此过程中, 为保证受试者护理过程的感受, 机器人与人背人过程一样具有前倾与升抬的动作 过程。

\section{2 被护理人舒适性测试}

被护理人在移乘过程中与机器人接触部位的受 力大小是影响其舒适的直接因素。由动力学分析与 仿真可知, 移乘过程中的被护理人胸部压力与腋下 的压力基本呈相反的变化趋势，且随胸靠的姿态的 改变而变化。因此, 在机器人胸靠与被护理人接触 部位安置阵列式柔性触觉传感器(TEKSCEN), 记录 8 名试验对象移乘过程中的胸部压力变化, 分析被 护理人的 “舒适” 与胸部压力之间的关系, 以便于 机器人动作的设计。

试验之初, 试验对象结合自己身高及主观感受 调整発子高度及位置, 随后结合每位试验对象的主 观感受分别为其设计机器人的运动轨迹。根据机器 人的动作模式, 将机器人的搬运过程分为搬运起始 (被护理人即将离开登子)、搬运上升、上升与下降 转换点、搬运下降、慰部支撑(被护理人坐于机器人 臀部支撑) 5 个过程。依据每位试验对象的主观感受， 选取每个过程中其感受 “舒适” 的特征点, 拟合出 分别各位试验对象感受 “舒适” 的搬运轨迹。在主 
观评价的过程中, 将试验对象的舒适度感受分为“难 受” (-2)、“不舒服” $(-1)$ 、“可接受” $(0)$ 、“较好” (1)、“舒适” (2)5 个等级, 利用调查问卷的方式进行 主观评价 ${ }^{[22-25]}$, 如表 4 所示。为避免试验对象的腹 部饱涨影响搬运动作的评价, 试验在上午进行, 且 要求试验对象日前不饮酒、充分休息, 试验开始前 两小时内禁止饮食。此外, 为避免由于连续试验引 发的身体疲劳对试验结果造成影响, 在每次进行搬 运姿态评价后, 要求试验对象休息 $5 \mathrm{~min}$, 方可进 行下一次的动作试验。

表 4 试验对象主观评价表

\begin{tabular}{lccccc}
\hline & 难受 & 不舒服 & 可接受 & 较好 & 舒适 \\
\hline 腋下压迫 & -2 & -1 & 0 & 1 & 2 \\
胸部压迫 & -2 & -1 & 0 & 1 & 2 \\
腹部压迫 & -2 & -1 & 0 & 1 & 2 \\
总体评价 & -2 & -1 & 0 & 1 & 2 \\
\hline
\end{tabular}

以机器人动臂 1 关节为坐标原点, 根据每试验 对象的主观感受所设计的机器人的胸靠最高点运动 轨迹如图 16 所示。为确保移乘过程中试验对象的舒 适, 机器人的移乘轨迹随试验对象身高体重的不同 而存在差异。其中, 身高/体重比值相近的试验对象 由于身高的不同, 其移乘轨迹起始点的位置也存在 差异。随着试验对象身高的增加, 动作轨迹曲线呈 整体抬高趋势; 轨迹起始点横坐标值趋向减小, 纵 坐标值增加, 起抱位置更靠近机器人。同时, 身高 相近的试验对象由于体重差异, 其轨迹也存在区别, 随体重的增加, 其轨迹起始点的横坐标值增大, 起 抱位置远离机器人。

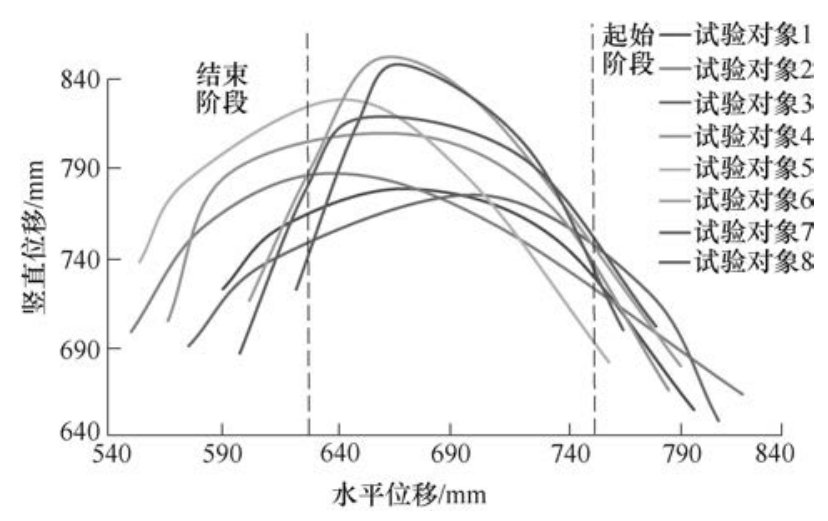

图 16 不同试验对象的机器人轨迹

根据试验对象的主观感受所设计的机器人轨迹 可完成对其安全舒适的移乘。在此基础上, 分析被 护理人胸部压力/压力中心与 “舒适” 之间的关系。 试验表明, 随着机器人胸靠姿态的改变, 试验对象
的胸部受力发生变化直接影响其主观感受。当移乘 过程中胸靠趋向坚直方向转动时, 试验对象会产 生因腋下受力过大而产生麻木甚至疼痛; 而当胸 靠趋向水平方向转动时, 试验对象的腋下压力减 小, 但随胸腹部压力的增大, 会出现呼吸受阻以 及腹部应力集中而导致身体不适。通过比较每位 试验对象在所选取的 5 个状态下的压力变化(表 5) 发现: 该机器人可通过胸靠的姿态调整实现对不 同身高体重的试验对象舒适的移乘; 且在机器人 将试验对象移乘至机器人臀部支撑的过程中, 当 试验对象胸部压力中心在距样机胸靠下边缘约 $1 / 3$ 处时, 试验对象不存在明显的腋下、胸部、腹 部受力过大以及接触部位应力集中的情况, 并在 此位姿下感受 “舒适”。通过对每位试验对象的单 独分析, 该位置基本对映每位试验对象的胸腹连 接区域; 当试验对象被移乘至机器人臀部支撑上 时, 由于试验对象下肢重量负担于机器人臀部支 撑上, 胸部负担减小, 压力中心位置向上移动基 本位于整个接触区域的中心。在此需要指出的是, 由于试验对象的身高体重存在差异性, 其压力中 心点位置以及其对映试验对象身体的详细位置稍 有区别。

表 5 不同试验对象胸部接触压力

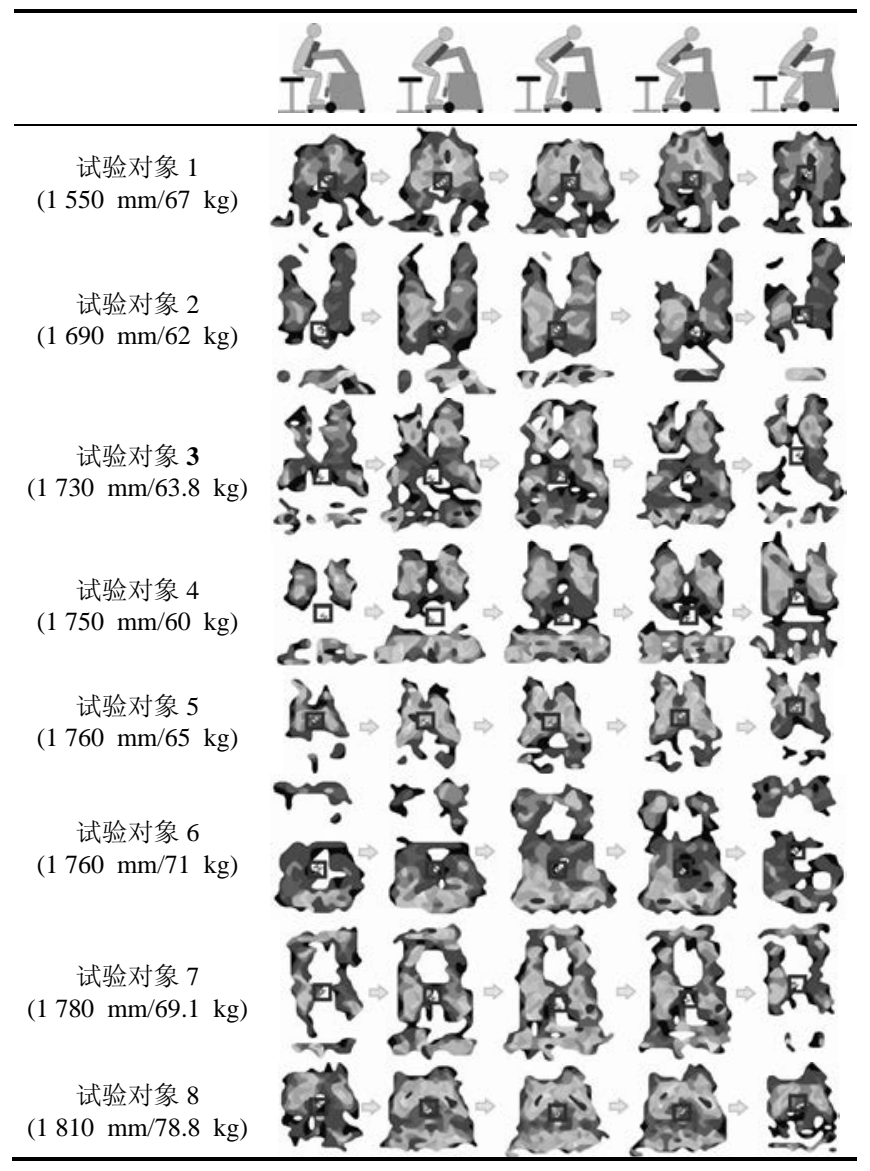




\section{6 结论}

本研究从人背人的运动特性入手, 研制了一款 仿人背抱式移乘护理机器人, 以满足下肢失能老人 的移乘需求, 减轻护理人员的身体负担。该机器人 采用 3 自由胸靠支撑被护理人的胸部, 模仿人背人 的动作, 实现被护理人在床、轮椅、则所马桶等生 活器具间的移乘服务。

本研究通过分析人背人的运动特征及影响移乘 舒适性的因素, 设计了机器人的构型, 建立了人机 系统力学模型, 并利用运动学与动力学解析, 优化 了运动轨迹, 使机器人具有合理的工作空间和运动 模式, 实现了机器人的仿人背抱动作。对 8 名不同 身高体重的试验对象的试验结果显示, 所研制的机 器人可以较为安全舒适地完成仿人背抱动作并在不 同生活器具间进行移乘服务。此外, 人机之间的相 互作用力的大小是影响被护理人舒适感的直接因 素, 该机器人可以通过调整胸靠的运动轨迹, 实现 不同身高体重的被护理人的舒适移乘，具有较高的 实用性。

在下一步的研究工作中, 将优化现有样机结构, 研究机器人对被护理人的姿态识别以及自适应动作 轨迹调整等方法，提升该机器人的整体性能。

\section{参 考 文 献}

[1] SHI Di, ZHANG Wuxiang, ZHANG Wei, et al. A review on lower limb rehabilitation exoskeleton robots[J]. Chinese Journal of Mechanical Engineering, 2019， 32(1): 74.

[2] SEFCIK J S, JOHNSON M J, YIM M, et al. Stakeholders' perceptions sought to inform the development of a low-cost mobile robot for older adults: A qualitative descriptive study[J]. Clinical Nursing Research, 2018, 27(1): 61-80.

[3] ZSIGA K, TÓTH A, PILISSY T, et al. Evaluation of a companion robot based on field tests with single older adults in their homes[J]. Assistive Technology, 2017: 1-8.

[4] 袁泉, 罗雪燕, 姚文兵. 失能老年人机构长期照护需求 及影响因素 $[\mathrm{J}]$. 中国老年学杂志, 2017, 37(24): 6214-6216.

YUAN Quan, LUO Xueyan, YAO Wenbing. Long-term care demands and influencing factors of disabled elderly institutions[J]. Chinese Journal of Gerontology, 2017, 37(24): 6214-6216.

[5] 王宝莲, 庞书勤, 陈芳, 等. 社区高龄老人家属照顾负
担现状及影响因素调查[J]. 护理研究, 2017, 31(28): 3520-3523.

WANG Baolian, PANG Shuqin, CHEN Fang, et al. Survey of status quo and influencing factors of family member's nursing burden on the community oldest-old[J] . Chinese Nursing Research, 2017, 31(28): 3520-3523.

[6] 杨帅, 曾佳琪, 王丽倩, 等. 护士职业性肌肉骨骼损伤 的研究进展 $[\mathrm{J}]$. 中国护理管理, 2018, 18(4): 89-93.

YANG Shuai, Zeng Jiaqi, WANG Liqian, et al. Research progress of occupational musculoskeletal injury among nurses[J]. Chinese Nursing Management, 2018, 18(4): 89-93.

[7] KUME Y, TSUKADA S, KAWAKAMI H. Design and evaluation of rise assisting bed “Resyone ${ }^{\circledR}$ ” based on ISO 13482[J]. Journal of the Robotics Society of Japan, 2015, 33(10): 781-788.

[8] 周思源, 张秋菊, 阴贺生. 床椅一体化护理床抬背机构 运动分析与滑移补偿 [J]. 机械传动, 2018, 42(9): 90-96. ZHOU Siyuan, ZHANG Qiuju, YIN Hesheng. Slippage compensation and movement analysis of carry back mechanism of the care bed combined with bed and chair[J]. Journal of Mechanical Transmission, 2018, 42(9): $90-96$

[9] FAUCHER M, BRULOTTE D A. Patient/invalid lift with support line bearing power and data communications: United States, US9421140B2 [P]. 2016-08-23.

[10] 高峰, 郭为忠. 中国机器人的发展战略思考 $[\mathrm{J}]$. 机械工 程学报, 2016, 52(7): 1-5.

GAO Feng, GUO Weizhong. Thinking of the development strategy of robots in China[J]. Journal of Mechanical Engineering, 2016, 52(7): 1-5.

[11] 颜云辉, 徐靖, 陆志国, 等. 仿人服务机器人发展与研 究现状 $[J]$. 机器人, 2017, 39(4): 551-564.

YAN Yunhui, XU Jing, LU Zhiguo. Development and research status of humanoid service robots[J]. Robot, 2017, 39(4): 551-564

[12] KAWAKAMI H, KUME Y, NAKAMURA $\mathrm{T}$, et al. Development of transfer assist robot[J]. Japan Society of Robotics, 2009, 188: 53-58.

[13] ODASHIMA T, ONISHI, M, TAHARA K, et al. Development and evaluation of a human-interactive robot platform “RI-MAN" [J]. Journal of the Robotics Society of Japan， 2007， 25(4): 554-565

[14] MUKAI $\mathrm{T}$, HIRANO $\mathrm{S}$, NAKASHIMA $\mathrm{H}$, et al. Development of a nursing-care assistant robot RIBA that 
can lift a human in its arms[C]// 2010 EEE/RSJ International Conference on Intelligent Robots and Systems, Oct 18-22, 2010, Taipei, Taiwan, China: IEEE, 2010: 5996-6001.

[15] DING Ming, MATSUBARA T, FUNAKI $\mathrm{Y}$, et al. Generation of comfortable lifting motion for a human transfer assistant robot[J]. International Journal of Intelligent Robotics and Applications, 2017, 1(1): 74-85.

[16] DING Jienan, LIM Y J, SOLANO M, et al. Giving patients a lift - the robotic nursing assistant (RoNA)[C]// 2014 IEEE International Conference on Technologies for Practical Robot Applications (TePRA), Apr. 14-15, 2014, Woburn, MA, USA: IEEE, 2014: 1-5.

[17] Toyota motor Co., Ltd. A patient transfer assist robot [EB/OL]. [2017-01-24]. http: // www.toyota.com.cn/ techn-ology /robo-t/robot04.php.

[18] Fuji Machinery Co., Ltd. Mobility support robot [EB/OL]. [2018-12-13]. https: //www.fuji.co.jp/en/about/hug/.

[19] ISO/IEC FCD 19774: 200x, Humanoid Animation, Annex B, Feature Points for the Human Body [EB/OL]. [2019-02-15]. https: //www.web3d.org/.

[20] CAO Yingyu, XUE Long, QI Bojin, et al. Composite configuration interventional therapy robot for the microwave ablation of liver tumors[J]. Chinese Journal of Mechanical Engineering, 2017, 30(6): 1416-1425.

[21] LEI Jingtao, YU Huangying, WANG Tianmiao. Dynamic bending of bionic flexible body driven by pneumatic artificial muscles (PAMs) for spinning gait of quadruped robot[J]. Chinese Journal of Mechanical Engineering, 2016, 30(1): 11-20.

[22] WANG Tianyi, JEONG H, WATANABE M, et al. Fault classification with discriminant analysis during sit-tostand movement assisted by a nursing care robot[J]. Mechanical Systems and Signal Processing, 2018, 113: 90-101.

[23] GONCALVES R S, HAMILTON T, DAHER A R, et al. MIT-Skywalker: Evaluating comfort of bicycle/saddle seat[C]// 2017 International Conference on Rehabilitation Robotics (ICORR), July 17-20, 2017, London, UK: IEEE, 2017: 516-520.

[24] MASTRIGT H V, GROENESTEIJN L, VINK P, et al. Predicting passenger seat comfort and discomfort on the basis of human, context and seat characteristics: A literature review[J]. Ergonomics, 2016, 60(7): 1-44.

[25] SATO S, GUO Shijie, INADA S, et al. Design of transfer motion and verification experiment of care assistant robot RIBA-II[J]. Transactions of the Japan Society of Mechanical Engineers Series C, 2012, 78(789): 595-608.

作者简介: 刘玉秀男, 1992 年出生, 博士研究生。主要研究方向为智 能护理机器人。

E-mail: liuyuxin654@163.com

郭士杰(通信作者), 男, 1963 年出生, 博士, 教授, 博士研究生导师。 主要研究方向为智能护理机器人、柔性步行助力机器人、无束缚生理信 息检测、机器人智能传感技术。

E-mail: guoshijie@hebut.edu.cn 